\title{
Towards Large-Size Ontology Alignment by Mapping Groups of Concepts
}

\author{
Patrice Buche ${ }^{1,3}$, Juliette Dibie-Barthélemy², and Liliana Ibănescu ${ }^{2}$ \\ 1 INRA - UMR IATE, 2, place Pierre Viala, F-34060 Montpellier Cedex 2, France \\ 2 INRA - Mét@risk \& AgroParisTech, 16 rue Claude Bernard, F-75231 Paris \\ Cedex 5, France \\ 3 LIRMM, CNRS-UM2, F-34392 Montpellier, France \\ Patrice.Buche@supagro.inra.fr, \\ $\{$ Juliette.Dibie, Liliana. Ibanescu\}@agroparistech.fr
}

\begin{abstract}
This paper presents a semi-automatic method to map groups of concepts between two ontologies.
\end{abstract}

\section{Mapping Groups of Concepts}

After a preliminary work presented in [1], we propose in this paper a new semiautomatic method to align large-size ontologies by reusing existing mappings between groups of concepts. Our method deals with ontologies, a reference one and a source one, which are composed of concepts, properties and axioms, where the axioms specify the range of properties for some concepts. The originality of our method is to map concepts not one-by-one, but groups by groups, using predefined mappings between groups of concepts and the concepts' descriptions by axioms. A group of concepts is defined by restrictions on properties. Given a new group of concepts in the reference ontology, the method finds first the "semantically close" groups to this new group, and then reuses the existing mappings to build the corresponding group of concepts in the source ontology.

Our approach is guided by the application needs and gives a first semiautomatic solution to make the work of the user easier when he/she has to align large-size and different ontologies in which concepts are described by many different properties. This method has been tested on a real application in food safety [2] and the first preliminary experimental results are promising.

\section{Related Works}

Ontology alignment is an active research area which has been largely studied in the literature (see 34]) and many systems have been developed to align ontologies (see the Ontology Alignment Evaluation Initiative 1 ). To the best of our knowledge, the problem of finding several to several correspondences between

${ }^{1}$ http://oaei.ontologymatching.org/ 
concepts has not been already addressed. Most work on ontology alignment studies how establishing one to one correspondences between concepts.

The problem of finding alignments between large-size ontologies has been especially addressed by works on ontology partition [56]. These works can be compared with ours since the groups of concepts can be considered as a kind of partition of the ontologies, but our method is guided by the existing groups and not by the alignment task, and, its goal is to build groups of concepts. The most close work to ours is the one of [7/8] which proposes to use Partial Reference Alignments (PRA) to partition the ontology into mappable groups. These PRA are subsets of all correct mappings and allow one to reduce the search space, to compute similarities between terms and to filter mapping suggestions. The main difference is that, in our case, the finality of our method is to find mappings between groups of concepts and not between concepts.

\section{Conclusion}

Our alignment method proposes a first step to map groups of concepts according to existing mappings between groups. The relationship which may exist in the reference ontology between a new group and the existing groups and their mappings plays an important role in the construction of the corresponding new group in the source ontology. Our method uses existing mappings to define new mappings which is an idea classically used in machine learning methods such as in the system GLUE 9]. We will study in which way we can use these methods to improve our algorithm.

\section{References}

1. Doussot, D., Buche, P., Dibie-Barthélemy, J., Haemmerlé, O.: Using Fuzzy Conceptual Graphs to Map Ontologies. In: Proceedings of ODBASE 2006. LNCS, vol. 4275, pp. 891-900. Springer, Heidelberg (2006)

2. Buche, P., Dibie-Barthélemy, J., Tressou, J.: Le logiciel CARAT, pp. 305-333. Lavoisier (2006)

3. Euzenat, J., Shvaiko, P.: Ontology Matching. Springer, Heidelberg (2007)

4. Noy, N.F.: Ontology Mapping, pp. 573-590. Springer, Heidelberg (2009)

5. Hu, W., Zhao, Y., Qu, Y.: Similarity-Based Ontology Alignment in OWL-Lite. In: Asian Semantic Web Conference, ASWC, pp. 72-83 (2006)

6. Hamdi, F., Safar, B., Zargayouna, H., Reynaud, C.: Partitionnement d'ontologies pour le passage l'échelle des techniques d'alignement. In: Extraction et Gestion des Connaissances, EGC, pp. 409-420 (2009)

7. Lambrix, P., Tan, H., Liu, Q.: SAMBO and SAMBOdtf Results for the Ontology Alignment Evaluation Initiative 2008. In: Sheth, A.P., Staab, S., Dean, M., Paolucci, M., Maynard, D., Finin, T., Thirunarayan, K. (eds.) ISWC 2008. LNCS, vol. 5318, Springer, Heidelberg (2008)

8. Lambrix, P., Liu, Q.: Using Partial Reference Alignments to Align Ontologies. In: Aroyo, L., Traverso, P., Ciravegna, F., Cimiano, P., Heath, T., Hyvönen, E., Mizoguchi, R., Oren, E., Sabou, M., Simperl, E. (eds.) ESWC 2009. LNCS, vol. 5554, pp. 188-202. Springer, Heidelberg (2009)

9. Doan, A., Madhavan, J., Dhamankar, R., Domingos, P., Halevy, A.Y.: Learning to match ontologies on the Semantic Web. Very Large Data Bases (VLDB) Journal 12(4), 303-319 (2003) 